\title{
Aplicabilidad de cuestionarios aplicados a pruebas sensoriales gastronómicas orientados al producto $\mathrm{y}$ al consumidor
}

\section{Applicability of questionnaires applied to sensorial gastronomic tests}

\author{
Diego Alfredo Salazar-Duque \\ Universidad Tecnológica Equinoccial, Ecuador
}

Autor para correspondencia: diegosalazarduque@hotmail.com

Fecha de recepción: 15 de marzo del 2019 - Fecha de aceptación: 31 de julio del 2019

Resumen: Antes de lanzar un producto al mercado, desde la óptica gastronómica, es necesario valorar si los distintos atributos que lo caracterizan son o no aceptados por la población. Para ello será fundamental realizar una evaluación sensorial a partir de las propiedades organolépticas que presenta dicho producto mediante la percepción generada con ayuda de los sentidos. El estudio tiene como objetivo principal proponer formatos prácticos que permitan evaluar ciertos criterios presentes en un producto culinario, tanto de forma analítica como afectiva. Como resultado final, se presentan dos instrumentos básicos, técnicos y de carácter metodológico, diseñados sobre una base bibliográfica, los cuales serán útiles para el desarrollo de pruebas gastronómicas; una orientada al producto y otra orientada al consumidor. Bajo esta dinámica, se concluye que, la existencia de una herramienta de análisis sensorial permitirá medir las diversas percepciones organolépticas de los alimentos que interactúan con los órganos de los sentidos clásicos (vista, olfato, gusto, tacto y auditivo), las cuales se encuentran interpretadas en el aspecto, olor, sabor, textura y sonido de un producto gastronómico.

Palabras Claves: propiedades organolépticas; atributos sensoriales; evaluación sensorial; gastronomía; consumidor

\begin{abstract}
Before launching a product to the market, from the gastronomic point of view, it is necessary to assess whether or not the different attributes that characterize it are accepted by the population. For this, it will be essential to carry out a sensory evaluation based on the organoleptic properties that this product presents through the perception generated with the help of the senses. This study aims to propose practical formats that allow the evaluation of certain criteria present in a culinary product, both analytically and affectively. As a final result, two basic instruments, technical and methodological in nature, are presented on a bibliographic basis, which are used for the development of gastronomic tests; a product orientation and another consumer orientation. Under this dynamic, it is concluded that, the existence of a sensory analysis tool will allow to measure the various organoleptic perceptions of foods that interact with the organs of the classical senses (sight, smell, taste, touch and auditory), which are found interpreted in the appearance, smell, taste, texture and sound of a gastronomic product.
\end{abstract}

Key Words: organoleptic properties; sensory sensor; sensory evaluation; gastronomy; consumer 


\section{Introducción}

En la actualidad, se pueden encontrar cientos, por no decir miles, de cuestionarios físicos o digitales que se han construido a lo largo del tiempo con el fin de levantar y medir un sinnúmero de indicadores referentes a diversas variables cuantitativas como cualitativas descritas según las necesidades del investigador, las cuales han sido previamente identificadas y definidas sobre una base teórica y metodológica estructurada referente a algún tema de investigación u objeto de estudio que requiere ser solucionado o considerado por su importancia, y del cual se desean obtener datos relevantes que apoyen a la posterior toma de decisiones que demande dicha investigación. Algunos de estos instrumentos pueden estar compuestos por sencillos o múltiples cuestionamientos que recolectan información necesaria, pertinente y actualizada.

De estos cuestionarios, el que más se destaca en una investigación descriptiva de carácter cuantitativo es la encuesta, un tipo de instrumento que recopila datos del entorno en donde se genera el estudio, el cual es llenado o contestado por diversas personas denominadas también sujetos de estudios, elementos de análisis o unidades de muestreo, de forma individual o grupal (autoadministrada o con presencia del encuestador). Para ello, aquellos sujetos han sido previamente definidos según el perfil que requiere la investigación y contribuyen con información fundamental para cumplir diversos objetivos delimitados según la necesidad de cada estudio (Hernández Sampieri, Fernández y Baptista, 2014).

Gracias a este tipo de cuestionario, y desde la investigación de mercados, se puede levantar información concerniente a diversas áreas o campos de estudio que requieren ser analizados y evaluados. Uno de estos es la percepción del comprador tanto sobre la oferta como sobre la demanda de productos y servicios existentes en el mercado. Es indudable que para este proceso es imperativo contar con instrumentos de recolección de información que tengan una validez y aplicabilidad para la formalidad de cada estudio. Desde un enfoque gastronómico, el desarrollo adecuado de un instrumento (cuestionario) en el que se apliquen criterios sensoriales contribuirá a un análisis más específico para evaluar productos culinarios. Desde la perspectiva de investigación, el nombre que se le da a esa metodología se denomina "panel de evaluación sensorial" (Hernández, 2005).

Preparaciones como sopas, platos principales a base de carne o pasta, bebidas a base de frutas, postres, entre otros, requieren ser evaluados a partir de sus atributos sensoriales mucho antes de ser ofertados al mercado para que no generen complicaciones futuras; más que todo, gracias a ello, se pueden mejorar sus características constantemente. Aquí, la evaluación de alimentos está determinada por los sentidos (Watts et al., 1992) y, por lo tanto, la existencia de una herramienta, que agrupe todos aquellos elementos indispensables e importantes que puedan ser útiles y didácticos para el encuestador (investigadores, profesionales o estudiantes pertenecientes a la rama de la gastronomía), es imperativo al momento de levantar datos mediante pruebas cuantitativas de consumo (Ramírez-Navas, 2012). Un documento con estas características podrá disminuir de una forma considerable algún posible sesgo que pueda existir durante la interpretación del cuestionario (Hernández Sampieri, Fernández y Baptista, 2014).

Sobre la base de los antecedentes antes mencionados, se ha identificado que una de las principales complicaciones que se pueden encontrar en la investigación de mercados es la 
consolidación de un cuestionario que sea útil para un panel de evaluación sensorial; un instrumento válido y de aplicabilidad en el sector gastronómico, sobre todo para aquellos estudios relacionados con la producción de platos culinarios tanto para alimentos como para bebidas de consumo inmediato.

A lo mencionado anteriormente, y como objetivo principal de este estudio, se considera establecer y proponer herramientas técnicas (cuestionarios) que permitan evaluar diversos atributos sensoriales o técnicos de alimentos o preparaciones gastronómicas (no aplicable a bebidas: cerveza, vinos, cocteles, gaseosas), que estén orientadas al producto y al consumidor en cuanto a preferencias, aceptabilidad o grado en que gusta un producto. Estas herramientas, sustentadas teóricamente en cuanto a su diseño (forma) y contenido (fondo), serán una técnica aplicable para ejecutar el panel de evaluación sensorial.

\section{Pruebas orientadas al producto y al consumidor}

Con el fin de aplicar un panel de evaluación sensorial, se requieren primero de ciertos criterios que son necesarios tomar en cuenta antes de ponerlo en marcha; para ello es necesario identificar qué métodos son los más adecuados para evaluar un producto o servicio.

De acuerdo a Ramírez-Navas (2012), las pruebas empleadas para evaluar la preferencia, aceptabilidad o grado en que gusta o disgusta un producto o un servicio, se las conoce como pruebas cuantitativas de consumo, las cuales se pueden desarrollar mediante el empleo de un cuestionario a partir de tres metodologías: mediante escalas, pruebas de preferencia, y de aceptabilidad o hedónica. De igual forma, también pueden considerarse pruebas complementarias de carácter cualitativo que manejen métodos como los grupos focales y la entrevista. Todos estos métodos contribuyen a obtener información sobre las percepciones del producto por parte del consumidor.

Con relación a las pruebas cuantitativas, entre las "escalas" que pueden ser consideradas en una investigación, según Guil (2006) y Hernández Sampieri, Fernández y Baptista (2014), se encuentran las diferenciales o de Thurstone (una respuesta de entre dos posibles opciones: "de acuerdo" o "desacuerdo"); las sumativas o de Likert (una respuesta de entre tres, cinco, siete o nueve puntos, o posibles opciones de respuestas según su puntuación) (Fermín et al., 2009; Guerra et al., 2011; Estrada et al., 2017; Barbosa-Martín et al., 2018;); las acumulativas o Guttman (valoración según el escalograma de actitudes), y el diferencial semántico (una sola calificación entre dos adjetivos polares de hasta siete posiciones), las cuales van acompañados de una serie de ítems o preguntas.

Con el fin de conocer la inclinación que se tiene sobre uno u otro producto, la técnica conocida como "pruebas de preferencia" es la metodología más adecuada para este proceso. Para esto, es necesario el manejo de dos o más muestras, las cuales son consideradas para ser evaluadas y comparadas entre sí (Ramírez-Navas, 2012), y en las que debe ser seleccionada la mejor alternativa de entre todas las distintas opciones.

Como tercera técnica cuantitativa orientada a evaluar la percepción del consumidor, se encuentra la "prueba de aceptabilidad" (prueba hedónica) (Palmero et al., 2010; Ramírez-Navas, 
2012; López-Ortiz, 2015), cuyo fin es evaluar los diversos atributos que pueden presentar un producto o servicio. Por lo general, en el sector de alimentos y bebidas, se comparan ciertos parámetros sensoriales a partir de las percepciones que se generan en el individuo, las cuales son medidas a través de los sentidos, aplicando escalas hedónicas de hasta nueve puntos (Likert).

Para este tipo de metodologías, las pruebas que se orienten tanto al consumidor como al producto pueden ser de carácter afectivo o analítico. Las primeras, afectivas, al reflejar resultados subjetivos (aceptabilidad de consumo de producto), dejan de lado aspectos técnicos que pueden no ser considerados en la evaluación de un producto gastronómico, donde el nivel, profundidad o calidad de respuesta dependerán del perfil del sujeto que llena el cuestionario. Por lo general, en referencia a la primera prueba, el cuestionario lo llena el consumidor del cual se espera obtener opiniones de agrado, preferencia o aceptabilidad de forma hedónica (López-Ortiz, 2015).

Con relación a la segunda prueba, analíticas, y con el fin de evitar subjetividad, se desarrollan evaluaciones sensoriales analíticas de una forma más técnica, las cuales pueden ser discriminativas (productos percibidos de manera diferente) o descriptivas (diferencias sensoriales), lo que genera una objetividad en los resultados. Para este último proceso (descriptivas sensoriales), es necesario considerar panelistas con cierto nivel de experiencia o gente entrenada que evalúe la prueba en espacios con condiciones controladas, esto evitará posibles respuestas sesgadas y una dispersión de los datos después de procesar los resultados y emplear en los cuestionarios escalas y descriptores con adjetivos que no impliquen gusto o disgusto personal (López-Ortiz, 2015).

Finalmente, se encuentran las pruebas cualitativas de consumo. En ellas, el manejo de "grupos focales" (Ivankovich-Guillén y Araya-Quesada, 2011), en los que participan hasta un promedio de doce individuos (Ramírez-Navas, 2012), o las "entrevistas" permiten obtener y examinar diversas opiniones acerca de distintos atributos de un producto mediante un conversatorio a base de preguntas estructuradas o cuestionamientos planeados cuidadosamente; aquí, la ayuda de ciertos recursos como grabadoras o celulares permiten grabar el proceso (Hernández Sampieri, Fernández y Baptista, 2014) para su posterior análisis.

Independientemente de qué metodología se utilice, es necesario resaltar que para las pruebas orientadas hacia las preferencias del consumidor no es necesario emplear panelistas entrenados o seleccionados por su agudeza sensorial sino trabajar con aquel posible consumidor potencial al que va dirigido dicho producto; en el caso de las pruebas orientadas al producto, se deben considerar jueces expertos o sujetos entrenados que evalúen, a más de los atributos, el nivel técnico que se requiere (Watts et al., 1992). En definitiva, un panel de evaluación sensorial deberá tener una estructura coherente y adecuada para el tipo de producto que se está tratando de evaluar, en este caso, un producto gastronómico y, para ello, será necesario delimitar la percepción sensorial a partir de sus atributos.

\section{Percepción sensorial (propiedades organolépticas)}

La percepción sensorial es la capacidad de captar a través de los sentidos las características de un producto alimenticio y sus resultados pueden ser presentados a través de 
datos sensoriales. Esto se genera por las propiedades organolépticas que poseen los alimentos y que son percibidas por los distintos sentidos. Entre las características organolépticas que se deben considerar para evaluar a nivel sensorial se encuentran: el aspecto (apariencia o forma), el sabor, la textura, el olor (Utset, 2007; Fermín et al., 2009; Guerra et al., 2011; López-Ortiz, 2015) y el sonido.

Para evaluar las propiedades organolépticas que posee un alimento, percibidas través de los sentidos, se requiere de un análisis sensorial o de un panel de evaluación sensorial en el que se identifiquen todas aquellas impresiones (sensograma) que tiene el consumidor o el evaluador sobre un producto (Sancho, Bota y Castro, 1999). Según Lawless y Heymann (2010) y RamírezNavas (2012), el análisis sensorial es una disciplina científica que permite medir, analizar e interpretar las distintas reacciones que presentan ciertos alimentos percibidos por los sentidos de la vista, olfato, gusto, tacto y oído. Esto permite controlar la calidad de un producto gastronómico mediante parámetros objetivos y así poder entender esas sensaciones subjetivas que experimentan o experimentarán los consumidores.

Uno de los efectos que también se pueden considerar durante una evaluación sensorial es el flavor (aroma): una sensación compleja que se obtiene por la estimulación de los órganos de varios sentidos en la boca de forma simultánea y que incluye el gusto, el olfato y las sensaciones táctiles o dolorosas de frío o calor (somatosensorial) (Sancho, Bota y Castro, 1999; Hernández, 2005). Este efecto, por lo general, se percibe al consumir alimentos que tienen varias texturas e ingredientes con diversas temperaturas de forma sincrónica.

A nivel culinario, la percepción sensorial permitirá traducir las preferencias de aceptabilidad que tienen los consumidores sobre sus gustos o desagrados culinarios (RamírezNavas, 2012), con lo que se corrigen posibles errores o potencian ciertas propiedades organolépticas que favorecerán a futuras preparaciones gastronómicas.

En este punto, es recomendable destacar una rama muy poco conocida de la estadística, la cual establece diversas técnicas de recolección de datos y métodos estadísticos de análisis aplicados en la industria alimentaria o agroalimentaria: la sensometría, una ciencia que permite comprender la intervención de los sensores naturales (ojos, oídos, nariz) (Césari, Ventrera y Gámbaro, 2018) para encontrar diferencias entre los productos. Si bien en este estudio no se profundiza esta disciplina, abre nuevas líneas de investigación a futuro para el desarrollo de nuevos estudios bibliográficos o empíricos en los que se relaciona a esta rama con la gastronomía.

\section{Atributos sensoriales}

Los atributos sensoriales constituyen aquellos estímulos sobre los cuales se emitirá un resultado de la calidad de los alimentos a través de un mecanismo de percepción sensorial (Duarte-García, 2018) mediante propiedades organolépticas. Para cada una de estas percepciones existen diversos atributos que deben ser estimados para una evaluación sensorial (sensograma) (Sancho, Bota y Castro, 1999). 
De acuerdo a Utset (2007), el aspecto es una característica organoléptica que debe ser considerada al momento de evaluar una muestra. Entre los atributos que deben ser estimados, se encuentra la forma, el tamaño, la integridad, la superficie y el color.

En el sabor, por su parte, se resaltan diversos atributos que son identificados en distinto nivel, instancia y forma por un individuo; estos se encuentran al consumir algún alimento existente o preparación culinaria de la diversa de cocina que hay en el mundo entero; entre los sabores básicos se encuentran el ácido, el dulce, el salado, el amargo, el umami más aquellos sabores secundarios percibidos en la boca al consumir algún alimento: fermentado, afrutado, astringente, picante, metálico o rancio (Hernández, 2005). Existe un estudio por parte de científicos de la Universidad de Borgoña quienes manifiestan haber descubierto unas células receptoras en la lengua (nuevas papilas gustativas) que poseen un receptor encargado de transmitir el sabor graso (de los lípidos), aunque este estudio no es concluyente aún se encuentra en procesos de debate (Laugerette et al., 2005) de ser así podría ser parte de este grupo de sabores.

Como las percepciones se producen en un órgano de los sentidos, la sensibilidad sensorial del tacto de un alimento se puede percibir en la piel y en la lengua, las cuales detectan la textura, el tamaño, la forma, la viscosidad, la adhesividad, la untuosidad, la dureza, etc. (Hernández, 2005). La textura, según Kramer (1964) referido por Hernández (2005), se genera a través de tres atributos: mecánicos, geométricos y de composición; cada uno de estos se subdivide en varias sensaciones. Por mencionar algunas, se encuentran: para el atributo mecánico, la dureza o la fracturabilidad; a nivel geométrico, la fibrosidad o esponjosidad; así, consecutivamente para cada propiedad (Utset, 2007; Hernández Sampieri, Fernández y Baptista, 2014) (Ver Tabla 1). Desde la óptica gastronómica, el tacto, conjuntamente con el sabor, pueden ser los atributos sensoriales más significativos e importantes que se encuentran directamente relacionados con el consumo de un alimento; de estos dependerá el éxito de un producto gastronómico ante otro.

En cuanto al olor, Castro, Ramanathan y Chennubhotla (2013), mediante un gran estudio técnico, indican que los olores se pueden clasificar en diez categorías básicas; ocho agradables: fragancia floral, fragancia leñosa o resinosa, frutal no cítrico, olor químico, mentolado o refrescante, dulce, quemado o ahumado, cítricos; más dos tipos de hedores nauseabundos: descompuesto y rancio. Con ayuda de esta categorización, se puede evaluar de forma más holística a nivel olfativo.

El sonido, por su parte, es una percepción poco evaluada o considerada por el consumidor; esto se debe a que no todas las preparaciones culinarias manifiestan sonidos por sí solas, a no ser que se encuentre dentro de la boca y se proceda a masticar e ingerir un alimento (sensación sonora) (Hernández, 2005). Por lo general, el ser humano tiene la capacidad de discriminar ciertos alimentos a partir de las peculiaridades sonoras que emite o que logra emitir ya sea producido en el ambiente o al momento de masticarlo. Como, por ejemplo, se puede resaltar el sonido que se genera al abrir una lata de bebida de alguna gaseosa al ser abierta o el sonido de un snack al ser mordido. Entre los atributos que pueden presentarse son crocante, crujiente o chirriante, o simplemente no presenta o no se percibe ningún sonido. 
Por otro lado, también existen aquellos atributos sensoriales que son necesarios considerar al momento de evaluar una bebida, cerveza o vino por ejemplo, los cuales requieren de ciertos parámetros sensoriales muy similares a los que se presentan en un alimento: el aroma, la apariencia, el sabor, la sensación en la boca o la astringencia (Strong y England, 2015), estos serán criterios a estimar para una valoración relacionada a una bebida. Para este estudio, no se contemplarán instrumentos de levantamiento de información destinados a este tipo de producto (bebidas).

\section{Evaluación gastronómica desde el enfoque técnico}

No solo la evaluación sensorial es indispensable al momento de lanzar un producto gastronómico al mercado, también es necesario considerar diversos aspectos técnicos que deben ser identificados antes de ser ofrecidos al consumidor. Estos aspectos deben ser evaluados por personas que saben del tema; es decir, técnicos o especialistas que se han formado para detectar ciertos aspectos que se relacionan con las percepciones sensoriales (chef, panaderos, pasteleros). Un postre, por ejemplo, puede ser agradable para algunos consumidores mientras que un pastelero especializado en la elaboración de ese producto podría juzgar su sabor o su elaboración de forma distinta; es decir, este especialista puede detectar qué técnicas aplicadas no fueron utilizadas correctamente o qué ingredientes no fueron manejados adecuadamente durante su elaboración. En definitiva, esto puede influir en las percepciones organolépticas durante su evaluación.

Otro criterio técnico que se debe evaluar es si las porciones presentadas en el producto gastronómico son adecuadas o, más aún, si se encuentran nutricionalmente balanceadas y, sobre todo, si se presentan estéticamente en un material (envase o empaque). Esto se debe a que ciertos consumidores relacionan las porciones con la calidad o el sabor; aquí, el nivel de percepción también puede ser influenciado por estos aspectos.

Finalmente, uno de los aspectos más relevantes e influyentes para el consumidor es la impresión general, la cual debe ser evaluada antes de que el producto sea comercializado. De considerar todos estos criterios, un proceso de evaluación sensorial podrá estar completa.

\section{Propuesta y proceso para el desarrollo de un Panel de Evaluación Sensorial}

Con el fin de evaluar una preparación gastronómica (alimento) que posteriormente se venderá en el mercado, es necesario primero establecer ciertos parámetros para el desarrollo de pruebas sensoriales orientadas al producto o al consumidor, las cuales puedan ser aplicadas en un panel de evaluación sensorial (Hernández, 2005), considerando ciertos aspectos técnicos de una forma más estructurada o sistematizada. Entre los criterios que se deben considerar están:

- Un moderador que explique la importancia de las pruebas sensoriales y cómo se va a desarrollar dicho proceso (Watts et al., 1992). Esta persona deberá tener una fluidez de palabra y conocer todos los aspectos referentes a la elaboración, presentación y distribución del producto que se desea orientar al consumidor. Junto a esta persona, deberá existir un equipo que colabore en elaborar y repartir el producto gastronómico, denominado para este punto como la "muestra" entre los panelistas, y los materiales 
necesarios que faciliten el proceso de evaluación; posteriormente, este mismo equipo apoyará en retirar los residuos o materiales innecesarios para el desarrollo de la siguiente prueba.

- Un lugar físico que proporcione las condiciones adecuadas para desarrollar el proceso de evaluación (Watts et al., 1992; Hernández, 2005). Este espacio deberá estar controlado (López-Ortiz, 2015), de preferencia con ayuda de cabinas que separen a un individuo de otro, y equipado con todos los recursos necesarios para la comodidad del panelista que va a evaluar el producto. De ser un producto gastronómico a evaluar, el espacio podrá contener mesas, sillas, cubiertos, vasos, entre otros materiales indispensables para el éxito de la prueba.

- Un grupo de panelistas, evaluadores o jueces que estimarán el nivel de cada atributo del producto a medir. Pueden ser expertos en la elaboración de alimentos gastronómicos (posiblemente instructores en gastronomía), personas entrenadas con conocimientos básicos del tema (posiblemente estudiantes de gastronomía) o consumidores potenciales (Hernández, 2005). Para esto, se debe estimar un panel de 10 a 20 jueces de ambos sexos y de diversas edades (Watts et al., 1992; Pacheco-Delahaye y Testa, 2005; López-Ortiz, 2015), aunque no se elimina la posibilidad de aplicar a un número mayor de panelistas; no obstante, considerar muchos evaluadores puede ser una problema muy complejo debido a la gran cantidad de recursos que se requieren para su efecto. Es conveniente obtener opiniones individuales por parte de los panelistas y, para ello, es aconsejable evitar un intercambio de opinión o discusión entre los evaluadores seleccionados durante la prueba que pueda influir en los resultados.

- La muestra o las muestras, que se van a valorar deberán contar con su respectiva rotulación y explicación. De ser un producto gastronómico, es indispensable que mantenga la temperatura correspondiente con la que será ofertado y la textura adecuada (López-Ortiz, 2015) con la que se consumirá. A esto, se debe aumentar el material, recipiente o envase en donde se va a presentar, contener y ofertar la muestra siendo este un elemento importante durante la evaluación (Watts et al., 1992). Se recomienda que las muestras vayan acompañadas de una bebida adicional que permita ingerir los productos gastronómicos que van a ser evaluados y que no influya en el resultado sensorial final; para ello, el agua es el componente más adecuado. Se considera para el panel de evaluación sensorial manejar de dos a cinco muestras de productos gastronómicos con similares características, ingredientes y técnicas empleadas. Tres muestras es el número más aconsejable para el proceso de evaluación. Esto es con el fin de no sobrecargar de comida al juez o evaluador durante la alimentación, aunque esto también dependerá del tamaño de cada porción. Es necesario también establecer el orden de presentación de cada muestra durante su consumo tomando en consideración que ciertos ingredientes pueden alterar el sentido del gusto (López-Ortiz, 2015). Se recomienda realizar las pruebas una hora antes o dos horas después del almuerzo con el fin de que el panelista no sienta llenura por si ha consumido algún otro producto previo que pueda influir en el resultado (Watts et al., 1992). 
- Otros recursos indispensables que se deben estimar para el panel son una cámara de fotos que permita evidenciar el proceso durante la evaluación, un esferográfico y el cuestionario en el que se registrarán las percepciones sensoriales o técnicas con sus respectivas indicaciones y con los campos necesarios para ser contestados (Hernández, 2005). Con base en la literatura existente sobre diversas pruebas sensoriales, varios autores recomiendan manejar en un cuestionario una escala Likert de cinco puntos (Pacheco-Delahaye y Testa, 2005; Guil Bozal, 2006; Roque y Palacios, 2017) con valores de aceptación que permitan evaluar cada uno de los atributos (Sensometría) a nivel sensorial (prueba hedónica); esta es una escala sencilla de comprender y rápida de contestar para los panelistas (Osinski y Sánchez, 1998).

- Finalmente, una vez que se ha efectuado el panel de evaluación sensorial, será necesario codificar los datos y transferir a una matriz (Hernández Sampieri, Fernández y Baptista, 2014), el investigador deberá proceder a analizar (Watts et al., 1992) e interpretar los resultados. Con ello, se decidirá qué muestra es la que mejor aceptación tiene o sobre qué atributos se deben mejorar. Derndofer et al. (2005), referido por Fermín et al. (2009) y Ramírez-Navas (2012), mencionan que para determinar la habilidad de los panelistas, según el proceso discriminatorio realizado entre productos, se utiliza un ANOVA, el cual evalúa la importancia de uno o más factores al comparar las medias de la variable de respuesta en los diferentes niveles existentes. Es decir, obtener un p-valor por debajo del valor crítico establecido $(\mathrm{p}<0,05)$ para cada atributo específico por muestra quiere decir que existen diferencias significativas en las evaluaciones, es decir, no hay concordancia estadística en sus evaluaciones, mientras que, de no presentar diferencias significativas, se obtendrá un valor mayor al valor crítico establecido $(\mathrm{p}>0,05)$.

\section{Propuesta de cuestionarios aplicados al panel de evaluación sensorial}

Con el fin de levantar información sobre un producto gastronómico mediante una prueba sensorial analítica (técnica) por parte de expertos o panelistas entrenados (ver Tabla 1) o una prueba sensorial afectiva (no técnica) por parte del consumidor (ver Tabla 2), se proponen dos formatos (cuestionarios) útiles para un panel de evaluación sensorial, el cual puede ser modificado dependiendo de la necesidad. Entre las partes que se destacan en el cuestionario aplicado a pruebas sensoriales gastronómicas orientadas al producto o al consumidor están:

- Una parte donde se manifiesta los datos generales del especialista o juez que evaluará cada una de las propiedades organolépticas que posee cada muestra incluye nombre, fecha de evaluación, lugar que ocupa la muestra en la prueba (A, B, C, etc.) y el nombre genérico del producto. Datos importantes para una posterior consulta en el caso de tener resultados inesperados o para comprobar la consistencia de los resultados obtenidos. Este espacio deberá ser llenado previamente por el investigador. Es decir, se deberá aplicar un cuestionario (formato) por cada muestra utilizada y por panelista.

- Una segunda parte donde se manifiestan las indicaciones que el evaluador debe seguir detenidamente para aplicar y completar el cuestionario de forma adecuada y sin errores posteriormente. 
- Una tercera parte donde se evalúa cada uno de los atributos que posee la muestra mediante una prueba sensorial afectiva donde se establece una escala de medición de 5 puntos; no obstante, en los cuestionarios de análisis sensorial orientado al producto, se ha considerado también una valoración de 0 (cero) para aquellas evaluaciones donde posiblemente no se identifica o no percibe algún atributo (Tabla 2). De considerar a más de una prueba afectiva, o una prueba analítica, será necesario incorporar ciertos aspectos técnicos que complementen el proceso, los cuales dependerán de aquellos criterios indispensables para el desarrollo y distribución del producto gastronómico (Tabla 1).

- Finalmente, una parte donde se dispone de un espacio para llenar las observaciones que se tenga sobre la muestra evaluada.

\section{Validación de los instrumentos propuestos}

Para este estudio, y con el fin de validar estos instrumentos propuestos, se seleccionaron tres especialistas en la rama de la gastronomía que trabajan en establecimientos de alimentos y bebidas y que imparten cursos de cocina; tres personas con perfil de consumidor gastronómico que acuden frecuentemente a restaurantes y que conocen del ámbito gastronómico, y que debían manifestar las observaciones existentes sobre dichos cuestionarios y así poder realizar los ajustes necesarios que fortalezcan a su aplicabilidad.

En relación con las observaciones de los especialistas en la rama de la gastronomía sobre los cuestionarios de evaluación sensorial orientando al producto gastronómico (Tabla 1) se identificó que:

- Es necesario resaltar que el instrumento está enfocado a alimentos mas no o a bebidas, y por lo tanto es necesario manifestar en su encabezado que la evaluación sensorial está diseñada para este tipo de producto.

- Otra de las opiniones consideradas por los especialistas en gastronomía fue la propiedad organoléptica de sabor, en la cual consideraron que resaltan los cinco gustos básicos (ácido, dulce, salado amargo y umami) y separarlos de otros posibles sabores que también puedan ser percibidos y así evitar una confusión al momento de la evaluación. De igual forma, se contempla la omisión de la propiedad del sonido por ser un aspecto que puede causar inconvenientes al momento de evaluar por parte del consumidor.

- Por otro lado, se considera también incorporar como parte del cuestionario de evaluación sensorial, orientada al producto gastronómico, a la temperatura, por ser un criterio importante que debe poseer un alimento durante su oferta. Este atributo se lo incorpora en la sección de análisis sensorial como una sensación táctil de temperatura y como un criterio técnico de evaluación.

Con relación a la opinión emitida por los consumidores sobre los cuestionarios de evaluación sensorial orientando al consumidor gastronómico (Tabla 2) se identificó que: 
- Puede existir una confusión en ciertos términos y por lo tanto se debería redactar de una forma más coloquial. Para ello, se han cambiado dos palabras técnicas referentes a dos propiedades organolépticas (aspecto y olor) por dos términos más amigables (apariencia y aroma, respectivamente).

- Igual que con el instrumento orientado al producto, el cuestionario dedicado al consumidor deberá resaltar que su uso está enfocado a alimentos mas no a bebidas, y por lo tanto en necesario manifestar en su encabezado que la evaluación sensorial está diseñada para este tipo de producto.

Una vez corregidas las observaciones de los cuestionarios se los muestra para su aplicación.

Tabla 1. Instrumento de evaluación sensorial y técnico de un alimento orientado a un producto gastronómico

\section{CUESTIONARIO DE EVALUACIÓN SENSORIAL Y TÉCNICO DE UN ALIMENTO ORIENTADO AL PRODUCTO GASTRONÓMICO}

Saludos, el objetivo del presente estudio tiene como fin [redactar objetivo general del estudio]; para ello se le solicita nos colabore con un análisis sensorial sobre los productos gastronómicos que han sido seleccionados para esta prueba.

\section{DATOS GENERALES}

\begin{tabular}{|l|l|}
\hline $\begin{array}{l}\text { Nombre del evaluador } \\
\text { 1: }\end{array}$ & \\
\hline Fecha de evaluación: & \\
\hline Nombre del producto: & \\
\hline Muestra: & A \\
\hline
\end{tabular}

\section{INDICACIONES}

Frente a usted, se encuentran varias muestras de [redactar el nombre genérico, variedad o tipo de producto gastronómico], las cuales deben ser evaluadas según la intensidad que posee cada uno de sus atributos. Se le solicita marcar con una $\mathrm{X}$ el nivel de escala que usted considera que posee el producto siendo 5 el valor que más predomina.

\section{ANÁLISIS SENSORIAL}

\begin{tabular}{|c|c|c|c|c|c|c|c|c|c|c|c|c|c|c|c|}
\hline \multirow{2}{*}{$\begin{array}{c}\text { Propied } \\
\text { ades } \\
\text { organolép } \\
\text { ticas }\end{array}$} & \multirow{2}{*}{$\begin{array}{c}\text { Atributo } \\
\mathbf{s} \\
\text { sensoriales }\end{array}$} & \multicolumn{6}{|c|}{$\begin{array}{l}\text { Intensidad de } \\
\text { percepción }\end{array}$} & \multirow{2}{*}{$\begin{array}{c}\text { Propied } \\
\text { ades } \\
\text { organolép } \\
\text { ticas }\end{array}$} & \multirow{2}{*}{$\begin{array}{c}\text { Atrib } \\
\text { utos } \\
\text { sensoria } \\
\text { les }\end{array}$} & \multicolumn{6}{|c|}{$\begin{array}{l}\text { Intensidad de } \\
\text { percepción }\end{array}$} \\
\hline & & $\mathbf{0}$ & 1 & 2 & 3 & 4 & 5 & & & $\mathbf{0}$ & 1 & 2 & 3 & 4 & 5 \\
\hline \multirow{2}{*}{ Aspecto } & Color & & & & & & & \multirow{2}{*}{ Sonido } & $\begin{array}{l}\text { Sin } \\
\text { sonido }\end{array}$ & & & & & & \\
\hline & Brillo & & & & & & & & $\begin{array}{l}\text { Croca } \\
\text { nte }\end{array}$ & & & & & & \\
\hline
\end{tabular}




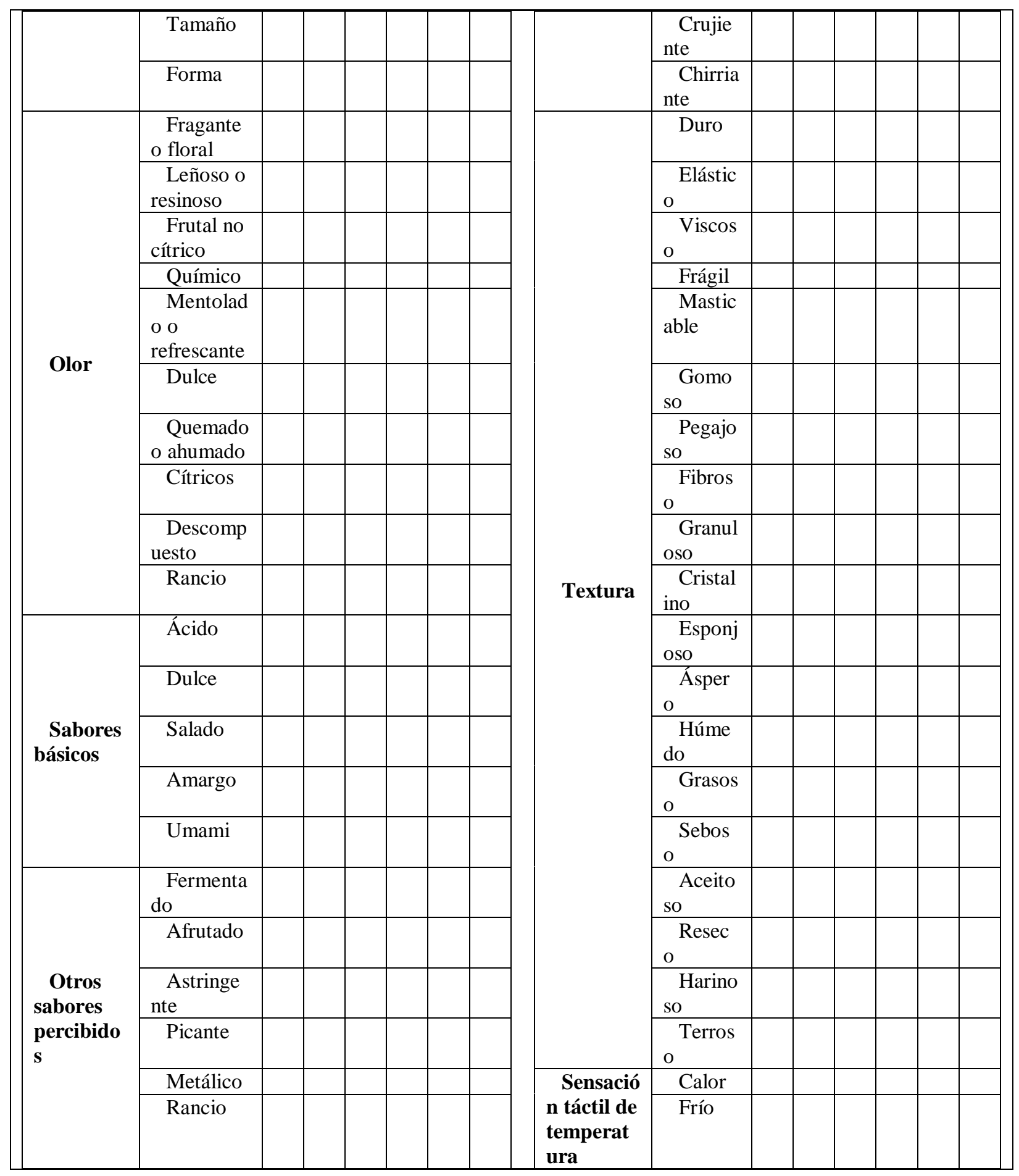




\begin{tabular}{|c|c|c|c|c|c|c|c|c|c|c|c|}
\hline \multicolumn{12}{|l|}{ ANÁLISIS TÉCNICO } \\
\hline CRITERIO TÉCNICOS & $\begin{array}{l}\text { Totalmente } \\
\text { en } \\
\text { desacuerdo }\end{array}$ & \multicolumn{2}{|c|}{$\begin{array}{c}\text { En } \\
\text { desacuerdo }\end{array}$} & \multicolumn{3}{|c|}{$\begin{array}{c}\text { Ni de } \\
\text { acuerdo, ni } \\
\text { en } \\
\text { desacuerdo }\end{array}$} & I & & \multicolumn{3}{|c|}{$\begin{array}{l}\text { Totalmente } \\
\text { de acuerdo }\end{array}$} \\
\hline \multicolumn{12}{|l|}{$\begin{array}{r}\text { Las técnicas empleadas en la } \\
\text { preparación contribuyen al } \\
\text { desarrollo del producto final } \\
\end{array}$} \\
\hline \multicolumn{12}{|l|}{$\begin{array}{r}\text { Las porciones consideradas } \\
\text { para la presentación del } \\
\text { producto gastronómico son } \\
\text { adecuadas }\end{array}$} \\
\hline \multicolumn{12}{|l|}{$\begin{array}{r}\text { El producto gastronómico se } \\
\text { encuentra nutricionalmente } \\
\text { balanceado }\end{array}$} \\
\hline \multicolumn{12}{|l|}{$\begin{array}{r}\text { La temperatura con la que se } \\
\text { presenta el plato gastronómico } \\
\text { es la adecuada }\end{array}$} \\
\hline \multicolumn{12}{|l|}{$\begin{array}{r}\text { El diseño del } \\
\text { plato/empaque/envase que lleva } \\
\text { el producto gastronómico } \\
\text { favorece la presentación }\end{array}$} \\
\hline \multicolumn{12}{|l|}{$\begin{array}{r}\text { El material empelado en el } \\
\text { plato/empaque/envase que lleva } \\
\text { el producto gastronómico es el } \\
\text { adecuado }\end{array}$} \\
\hline $\begin{array}{r}\text { La presentación final o } \\
\text { impresión general del producto } \\
\text { gastronómico influye en mi } \\
\text { antes de consumirlo }\end{array}$ & & & & & & & & & & & \\
\hline \multicolumn{12}{|c|}{ PRUEBA EVALUATIVA DE CONSUMO } \\
\hline \multicolumn{2}{|c|}{$\begin{array}{l}\text { Califique del } 1 \text { al } 10 \text { esta muestra donde } 1 \text { no } \\
\text { consumiría nunca este alimento y } 10 \text { donde sí } \\
\text { consumiría frecuentemente este alimento }\end{array}$} & sí & 2 & $\mathbf{3}$ & 4 & 5 & 6 & 7 & 8 & 9 & 10 \\
\hline \multicolumn{12}{|l|}{ OBSERVACIONES } \\
\hline & GRACIAS POR & $\mathrm{SU} \mathrm{CO}$ & LAB & RA & IÓN & & & & & & \\
\hline
\end{tabular}




\section{Conclusiones}

Al existir una gran diversidad de formatos para levantar información cuantitativa, pueden existir inconsistencias entre diversas investigaciones; ya sea porque cada investigador construye su propio instrumento a partir de su propio juicio, omitiendo algunos criterios importantes, o por desconocimiento de diversos atributos que pueden ser importantes para el estudio. Con base en esto, es imperativo proponer varios instrumentos que contribuyan a levantar ciertas percepciones sensoriales durante un panel de evaluación sensorial para productos gastronómicos (alimentos o bebidas) que se deseen ofrecer al mercado. Por lo tanto, es necesario diseñar cuestionarios tanto para alimentos como para bebidas.

Se considera que existen diversas percepciones organolépticas de los alimentos que interactúan con los órganos de los sentidos clásicos (vista, olfato, gusto, tacto y auditivo), interpretadas como aspecto, olor, sabor, textura y sonido. Cada alimento se manifiesta de distinta forma y el ser humano, a partir de su evaluación, mide el nivel de aceptabilidad a partir de sus gustos o desagrados culinarios.

Los instrumentos necesarios para evaluar la percepción sensorial de un alimento gastronómico deberán estar diseñadas tanto para pruebas orientadas al producto gastronómico como para pruebas orientadas al consumidor. Para las primeras pruebas, será necesario identificar un panel de expertos que, a más de medir los atributos sensoriales, deberá evaluar aspectos técnicos que son necesarios para la elaboración del producto o, posiblemente, aspectos administrativos como precio, publicidad, proceso de distribución, entre otros; mientras que para el segundo tipo de prueba es necesario identificar el consumidor potencial que va a adquirir el producto que será a futuro ofertado al mercado y del cual será evaluado a partir de su nivel de aceptación.

Gracias a este aporte, se generan las bases para futuras investigaciones académicas relacionadas con estudios sensoriales, comerciales o productivos, los cuales deberán aportar datos cuantitativos que fortalezcan estos instrumentos. De igual forma, también se identifica la necesidad de levantar, analizar, desarrollar o proponer cuestionarios aplicados a bebidas en los que se considere una evaluación sensorial aplicada al sector gastronómico a partir de sus atributos.

En el caso de modificar algún criterio importante de estos dos instrumentos se recomienda realizar una validación con algún especialista en el tema antes de aplicarlos.

\section{Bibliografía}

Barbosa-Martín, E. et al. (2018). Evaluación de la calidad de galletas reducidas en calorías endulzadas con hojas de stevia rebaudiana bertoni. Interciencia, 43(1), 17 - 22.

Castro, J. B., Ramanathan, A. y Chennubhotla, C. S. (2013). Categorical dimensions of human odor descriptor space revealed by non-negative matrix factorization. PloS one, 8(9), 1-16.

Césari, M., Ventrera, N. y Gámbaro, A. (2018). Análisis de datos sensoriales de tomate triturado con lógica difusa y técnicas multivariadas. Revista de la Facultad de Ciencias Agrarias, $50(1), 233-248$. 
Duarte-García, C. (2018). Valoración de atributos establecidos para controlar la calidad sensorial en alimentos. Ciencia y Tecnología de Alimentos, 27(2), 23-30.

Estrada, J. et al. (2017). Elaboración de pan artesanal utilizando harina de sorgo blanco (sorghum bicolor 1. moench) y trigo (triticum), adicionado con moringa (m. oleífera) para favorecer la calidad nutrimental. Jóvenes en la ciencia, 3(2), 60-64.

Fermín, N. et al. (2009). Entrenamiento sensorial para la evaluación de la calidad de un jamón endiablado. UDO Agrícola, 9(3), 640-652.

Guerra, M. A. et al. (2011). Efecto de la harina de plátano sobre la calidad de un embutido tipo mortadella. Ciencia y Tecnología de Alimentos, 21(3), 22 - 26.

Guil Bozal, M. (2006). Escala mixtaLikert-Thurstone. Anduli, 5, 81-95.

Hernández Sampieri, R., Fernández, C.y Baptista, P. (2014). Metodología de la Investigación (Vol. sexta edición). México D.F.: McGraw-Hill.

Hernández, E. (2005). Evaluación sensorial. Bogotá: Universidad Nacional Abierta y A distancia - UNAD.

Ivankovich-Guillén, C. y Araya-Quesada, Y. (2011). Focus groups: técnica de investigación cualitativa en investigación de mercados. Revista de Ciencias Económicas, 29(1), 545-554.

Laugerette, F. et al. (2005). CD36 involvement in orosensory detection of dietary lipids, spontaneous fat preference, and digestive secretions. The Journal of clinical investigation, 115(11), 3177-3184.

Lawless, H. T. y Heymann, H. (2010). Sensory evaluation of food: principles and practices. Springer Science \& Business Media.

López-Ortiz, N. C. (2015). La cuestión de las sensaciones gustativas básicas. Perspectivas en Nutrición Humana, 17(2), 185-194.

Osinski, I. y Sánchez, A. (1998). Categorías de respuesta en escalas tipo Likert. Psicothema, 10(3), 623-631.

Pacheco-Delahaye, E. y Testa, G. (2005). Evaluación nutricional, física y sensorial de panes de trigo y plátano verde. Interciencia, 30(5), 300-304.

Palmero, J. et al. (2010). Evaluación Sensorial de un Jamón Cocido Elaborado con Carnes de Chivo, Pollo y Cerdo. Revista Agrollania de Ciencia y Tecnología, 7, 25-31.

Ramírez-Navas. (2012). Análisis sensorial: pruebas orientadas al consumidor. ReCIteIa, 12(1), 84 $-102$.

Roque, A. y Palacios, G. (2017). Desarrollo y evaluación sensorial de dos productos de calabaza (Cucurbita pepo L. y Cucurbita moschata Poiret) Cucurbitaceae. Lacandonia, 7(2), 77-82.

Sancho, J., Bota, E. y Castro, J. (1999). Introducción al análisis sensorial. Barcelona: Edicions Universitat Barcelona.

Strong, G., \& England, K. (2015). Beer judge certification program. Beer Style Guidelines. Obtenido de https://www.bjcp.org/docs/2015_Guidelines_Beer.pdf

Utset, E. Z. (2007). Evaluación objetiva de la calidad sensorial de alimentos procesados. Ciudad de La Habana: Editorial Universitaria.

Watts, B. et al. (1992). Métodos sensoriales básicos para la evaluación de alimentos. Ottawa: International Development Research Centre. 